\title{
Periodic table for topological insulators and superconductors
}

\author{
Alexei Kitaev \\ California Institute of Technology, Pasadena, CA 91125, U.S.A.
}

\begin{abstract}
Gapped phases of noninteracting fermions, with and without charge conservation and time-reversal symmetry, are classified using Bott periodicity. The symmetry and spatial dimension determines a general universality class, which corresponds to one of the 2 types of complex and 8 types of real Clifford algebras. The phases within a given class are further characterized by a topological invariant, an element of some Abelian group that can be $0, \mathbb{Z}$, or $\mathbb{Z}_{2}$. The interface between two infinite phases with different topological numbers must carry some gapless mode. Topological properties of finite systems are described in terms of $K$-homology. This classification is robust with respect to disorder, provided electron states near the Fermi energy are absent or localized. In some cases (e.g., integer quantum Hall systems) the $K$-theoretic classification is stable to interactions, but a counterexample is also given.
\end{abstract}

Keywords: Topological phase, K-theory, K-homology, Clifford algebra, Bott periodicity

PACS: 73.43.-f, 72.25.Hg, 74.20.Rp, 67.30.H-, 02.40.Gh, 02.40.Re

The theoretical study $[1,2,3]$ and experimental observation [4] of the quantum spin Hall effect in 2D systems, followed by the discovery of a similar phenomenon is 3 dimensions $[5,6,8,10,11]$, have generated considerable interest in topological states of free electrons. Both kinds of systems are time-reversal invariant insulators. More specifically, they consist of (almost) noninteracting fermions with a gapped energy spectrum and have both the time-reversal symmetry $(T)$ and a $U(1)$ symmetry $(Q)$. The latter is related to the particle number, which is conserved in insulators but not in superconductors or superfluids. Topological phases with only one of those symmetries, or none, are also known. Such phases generally carry some gapless modes at the boundary. ${ }^{1}$

The classification of gapped free-fermion systems depends on the symmetry and spatial dimension. For example, two-dimensional insulators without $T$ symmetry are characterized by an integer $v$, the quantized Hall conductivity in units of $e^{2} / h$. For systems with discrete translational symmetry, it can be expressed in terms of the band structure (more exactly, the electron eigenstates as a function of momentum); such an expression is known as the TKNN invariant [13], or the first Chern number. A similar topological invariant (the $k$-th Chern number) can be defined for any even dimension $d$. For $d=0$, it is simply the number of single-particle states with negative energy $\left(E<E_{F}=0\right)$, which are filled with electrons.

However, the other three symmetry types (no symmetry, $T$ only, or both $T$ and $Q$ ) do not exhibit such a simple pattern. Let us consider systems with no symmetry at all. For $d=0$, there is a $\mathbb{Z}_{2}$ invariant: the number of elec-

\footnotetext{
${ }^{1}$ In contrast, strongly correlated topological phases (with anyons in the
} bulk) may not have gapless boundary modes[12]. trons $(\bmod 2)$ in the ground state. For $d=1$, a system in this symmetry class, dubbed "Majorana chain", also has a $\mathbb{Z}_{2}$ invariant, which indicates the presence of unpaired Majorana modes at the ends of the chain [14]. But for $d=2$ (e.g., a $p_{x}+i p_{y}$ superconductor), the topological number is an integer though an even-odd effect is also important $[15,16]$.

$T$-invariant insulators have an integer invariant (the number of particle-occupied Kramers doublet states) for $d=0$, no invariant for $d=1$, and a $\mathbb{Z}_{2}$ invariant for $d=2[1,2]$ and for $d=3[5,6,8]$. 3D crystals (i.e., systems with discrete translational symmetry) have an additional $3 \mathbb{Z}_{2}$ invariant, which distinguishes so-called "weak topological insulators".

With the exception just mentioned, the topological numbers are insensitive to disorder and can even be defined without the spectral gap assumption, provided the eigenstates are localized. This result has been established rigorously for integer quantum Hall systems [17, 18, 19], where the invariant $v$ is related to the index theory and can be expressed as a trace of a certain infinite operator, which represents the insertion of a magnetic flux quantum at an arbitrary point. Its trace can be calculated with sufficient precision by examining an $l$-neighborhood of that point, where $l$ is the localization length. A similar local expression for the $\mathbb{Z}_{2}$ invariant of a $1 D$ system with no symmetry has been derived in Appendix C of Ref. [16]; it involves an infinite Pfaffian or determinant.

In this paper, we do not look for analytic formulas for topological numbers, but rather enumerate all possible phases. Two Hamiltonians belong to the same phase if they can be continuously transformed one to the other while maintaining the energy gap or localization; we will elaborate on that later. The identity of a phase can be determined by some local probe. In particular, the Hamil- 
TABLE 1. Classification of free-fermion phases with all possible combinations of the particle number conservation $(Q)$ and time-reversal symmetry $(T)$. The $\pi_{0}\left(C_{q}\right)$ and $\pi_{0}\left(R_{q}\right)$ columns indicate the range of topological invariant. Examples of topologically nontrivial phases are shown in parentheses.

\begin{tabular}{|c|c|c|c|}
\hline$q$ & $\pi_{0}\left(C_{q}\right)$ & $d=1$ & $d=2$ \\
\hline 0 & $\mathbb{Z}$ & & (IQHE) \\
\hline 1 & 0 & & \\
\hline
\end{tabular}

Above: insulators without time-reversal symmetry (i.e., systems with $Q$ symmetry only) are classified using complex $K$ theory.

Right: superconductors/superfluids (systems with no symmetry or $T$-symmetry only) and time-reversal invariant insulators (systems with both $T$ and $Q$ ) are classified using real $K$-theory.

\begin{tabular}{ccccc}
\hline$q$ & $\pi_{0}\left(R_{q}\right)$ & $d=1$ & $d=2$ & $d=3$ \\
\hline 0 & $\mathbb{Z}$ & & no symmetry & $T$ only \\
& & no symmetry & $\left(p_{x}+i p_{y}\right.$, e.g., SrRu) & $\left({ }^{3} \mathrm{He}-B\right)$ \\
1 & $\mathbb{Z}_{2}$ & $($ Majorana chain) & $\left(\left(p_{x}+i p_{y}\right) \uparrow+\left(p_{x}-i p_{y}\right) \downarrow\right)$ & $T$ and $Q$ \\
& & $(\mathrm{BiSb})$ & \\
2 & $\mathbb{Z}_{2}$ & $\left((\mathrm{TMTSF})_{2} \mathrm{X}\right)$ & $T$ and $Q$ & \\
& & $(\mathrm{HgTe})$ & \\
3 & 0 & $T$ and $Q$ & & \\
4 & $\mathbb{Z}$ & & & no symmetry \\
5 & 0 & & & \\
6 & 0 & & & \\
7 & 0 & & & \\
\end{tabular}

tonian around a given point may be represented (in some non-canonical way) by a mass term that anticommutes with a certain Dirac operator; the problem is thus reduced to the classification of such mass terms.

Prior to this work, there have been several results toward unified classification of free-fermion phases. Altland and Zirnbauer [20] identified 10 symmetry classes of matrices, ${ }^{2}$ which can be used to build a free-fermion Hamiltonian as a second-order form in the annihilation and creation operators, $\hat{a}_{j}$ and $\hat{a}_{j}^{\dagger}$. The combinations of $T$ and $Q$ make 4 out of 10 possibilities. However, the symmetry alone is only sufficient to classify systems in dimension 0 . For $d=1$, one may consider a zero mode at the boundary and check whether the degeneracy is stable to perturbations. For example, an unpaired Majorana mode is stable. In higher dimensions, one may describe the boundary mode by a Dirac operator and likewise study its stability. This kind of analysis has been performed on a case-by-case basis and brought to completion in a recent paper by Schnyder, Ryu, Furusaki, and Ludwig [21]. Thus, all phases up to $d=3$ have been characterized, but the collection of results appears irregular.

A certain periodic pattern for $\mathbb{Z}_{2}$ topological insulators has been discovered by Qi, Hughes, and Zhang [22]. They use a Chern-Simons action in an extended space, which includes the space-time coordinates and some parameters. This approach suggests some operational interpretation of topological invariants and may even work for interacting systems, though this possibility has not been explored. In addition, the authors mention Clifford algebras, which play a key role in the present paper.

\footnotetext{
2 These classes are often associated with random matrix ensembles, but the symmetry pertains to concrete matrices rather than the probability measure.
}

We report a general classification scheme for gapped free-fermion phases in all dimensions, see Table 1. It actually consists of two tables. The small one means to represent the aforementioned alternation in TR-broken insulators (a unique trivial phase for odd $d$ vs. an integer invariant for even $d$ ). The large table shows a period 8 pattern for the other three combinations of $T$ and $Q$. Note that phases with the same symmetry line up diagonally, i.e., an increase in $d$ corresponds to a step up $(\bmod 8) .(T$-invariant $1 \mathrm{D}$ superconductors were studied in Ref. [9]. The $\left(p_{x}+i p_{y}\right) \uparrow+\left(p_{x}-i p_{y}\right) \downarrow$ phase was proposed in Refs. [23, 7, 21]; the last paper also describes an integer invariant for ${ }^{3} \mathrm{He}-B$.) The $2+8$ rows (indexed by q) may be identified with the Altland-Zirnbauer classes arranged in a certain order; they correspond to 2 types of complex Clifford algebras and 8 types of real Clifford algebras. Each type has an associated classifying space $C_{q}$ or $R_{q}$, see Table 2 . Connected components of that space (i.e., elements of $\pi_{0}\left(R_{q}\right)$ or $\pi_{0}\left(C_{q}\right)$ ) correspond to different phases. But higher homotopy groups also have physical meaning. For example, the theory predicts that $1 \mathrm{D}$ defects in a 3D TR-broken insulator are classified by $\pi_{1}\left(C_{1}\right)=\mathbb{Z}$.

The $(\bmod 2)$ and $(\bmod 8)$ patterns mentioned above are known as Bott periodicity; they are part of the mathematical subject called $K$-theory. It has been applied in string theory but not so much in condensed matter physics. One exception is Horava's work [24] on the classification of stable gapless spectra, i.e., Fermi surfaces, lines, and points. In this paper, we mostly use results from chapters II-III of Karoubi's book [25], in particular, the relation between the homotopy-theoretic and Clifford algebra versions of $K$-groups (a variant of the AtiyahBott-Shapiro construction [26]). 
TABLE 2. Bott periodicity in complex and real $K$-theory. (The parameters $k, m, n$ should be taken to infinity.)

\begin{tabular}{ccc}
\hline$q \bmod 2$ & Classifying space $C_{q}$ & $\pi_{0}\left(C_{q}\right)$ \\
\hline 0 & $(U(k+m) /(U(k) \times U(m))) \times \mathbb{Z}$ & $\mathbb{Z}$ \\
1 & $U(n)$ & 0
\end{tabular}

Above: The classifying space $C_{0}$ parametrizes Hermitian matrices $X$ with \pm 1 eigenvalues. $C_{q}$ is the $q$-th loop space of $C_{0}$; it parametrizes such matrices $X$ that anticommute with $q$ Clifford generators.

Right: Similar classification for real symmetric matrices.

\section{SOME EXAMPLES}

To get a glimpse of the mathematical structure underlying the topological classification, we consider a secondorder transition between two phases, where the energy gap vanishes at some value of parameters. In this case, the low-energy Fermi modes typically have a Dirac spectrum, and the phases differ by the sign of the mass term.

Let us begin with the simplest example, the Majorana chain [14]. This model has one spinless Fermi mode per site, but the number of particles is not conserved, which calls for the use of Majorana operators:

$$
\hat{c}_{2 j-1}=\hat{a}_{j}+\hat{a}_{j}^{\dagger}, \quad \hat{c}_{2 j}=\frac{\hat{a}_{j}-\hat{a}_{j}^{\dagger}}{i} \quad(j=1, \ldots, n) .
$$

By convention, operators acting in the the Fock space (as opposed to the mode space) are marked with a hat. The Majorana operators are Hermitian and satisfy the commutation relations $\hat{c}_{l} \hat{c}_{m}+\hat{c}_{m} \hat{c}_{l}=2 \delta_{l m}$; thus, $\hat{c}_{1}, \ldots, \hat{c}_{2 n}$ may be treated on equal footing. (But it is still good to remember that $\hat{c}_{2 j-1}$ and $\hat{c}_{2 j}$ belong to the same site $j$.) The advantage of the Majorana representation is that all model parameters are real numbers.

A general free-fermion Hamiltonian for nonconserved particles has this form:

$$
\hat{H}_{A}=\frac{i}{4} \sum_{j, k} A_{j k} \hat{c}_{j} \hat{c}_{k}
$$

where $A$ is a real skew-symmetric matrix of size $2 n$. The concrete model is this:

$$
\hat{H}=\frac{i}{2}\left(u \sum_{l=1}^{n} \hat{c}_{2 l-1} \hat{c}_{2 l}+v \sum_{l=1}^{n-1} \hat{c}_{2 l} \hat{c}_{2 l+1}\right) .
$$

At the transition between "the "trivial phase" $(|u|>|v|)$ and the "topological phase" $(|u|<|v|)$, there are two counterpropagating gapless modes. They may be represented by two continuous sets of Majorana operators, $\hat{\eta}_{j}(x)(j=1,2)$. The effective Hamiltonian near the transition point has this form:

$$
\hat{H}=\frac{i}{2} \int \hat{\eta}^{T}\left(\begin{array}{cc}
\partial & m \\
-m & -\partial
\end{array}\right) \hat{\eta} d x, \quad \hat{\eta}=\left(\begin{array}{l}
\hat{\eta}_{1} \\
\hat{\eta}_{2}
\end{array}\right),
$$

\begin{tabular}{ccc}
\hline$q \bmod 8$ & Classifying space $R_{q}$ & $\pi_{0}\left(R_{q}\right)$ \\
\hline 0 & $(O(k+m) /(O(k) \times O(m))) \times \mathbb{Z}$ & $\mathbb{Z}$ \\
1 & $O(n)$ & $\mathbb{Z}_{2}$ \\
2 & $O(2 n) / U(n)$ & $\mathbb{Z}_{2}$ \\
3 & $U(2 n) / S p(n)$ & 0 \\
4 & $(S p(k+m) /(S p(k) \times S p(m))) \times \mathbb{Z}$ & $\mathbb{Z}$ \\
5 & $S p(n)$ & 0 \\
6 & $S p(n) / U(n)$ & 0 \\
7 & $U(n) / O(n)$ & 0 \\
\hline
\end{tabular}

where $m \sim u-v$. Thus, we need to study the Dirac operator $D=\gamma \partial+M$, where $\gamma=\sigma^{z}$ and $M=m i \sigma^{y}$. If $m$ gradually varies in space and changes sign, e.g., $m(x)=-a x$, the Dirac operator has a localized null state, which corresponds to an unpaired Majorana mode in the second quantization picture. The existence of the true null state is a subtle property, but it has a simple semiclassical analogue: a continuous transition between a positive and a negative value of $m$ is impossible without closing the gap.

We now consider a model with two real fermions propagating in each direction, so that the mass term has more freedom. This situation occurs, for example, at the edge of a 2D topological insulator. A gap opens in a magnetic field or in close contact with a superconductor [27]. The Hamiltonian is as follows:

$$
\begin{gathered}
\hat{H}=\frac{i}{2} \int \hat{\eta}^{T}(\gamma \partial+M) \hat{\eta} d x, \quad \hat{\eta}=\left(\begin{array}{c}
\hat{\psi}_{\uparrow}+\hat{\psi}_{\uparrow}^{\dagger} \\
-i\left(\hat{\psi}_{\uparrow}-\hat{\psi}_{\uparrow}^{\dagger}\right) \\
\hat{\psi}_{\downarrow}+\hat{\psi}_{\downarrow}^{\dagger} \\
-i\left(\hat{\psi}_{\downarrow}-\hat{\psi}_{\downarrow}^{\dagger}\right)
\end{array}\right) \\
\gamma=\left(\begin{array}{cc}
I & 0 \\
0 & -I
\end{array}\right), \quad M=\left(\begin{array}{cc}
-h_{z}\left(i \sigma^{y}\right) & m \\
-m^{T} & h_{z}\left(i \sigma^{y}\right)
\end{array}\right), \\
m=-h_{x}\left(i \sigma^{y}\right)+h_{y} I-(\operatorname{Re} \Delta) \sigma^{x}-(\operatorname{Im} \Delta) \sigma^{z} .
\end{gathered}
$$

If $h_{z}=0$, the energy gap is given by the smallest singular value of $m$; it vanishes at the transition between the "magnetic" and "superconducting" phase as the function $\operatorname{det}(m)=h_{x}^{2}+h_{y}^{2}-|\Delta|^{2}$ passes through zero. The presence of $h_{z}$ complicates the matter, but if the spectrum is gapped, $h_{z}$ can be continuously tuned to zero without closing the gap. We will see that, in general, the mass term can be tuned to anticommute with $\gamma$, in which case $M$ consists of two off-diagonal blocks, $m$ and $-m^{T}$.

With $n$ modes propagating in each direction, the nondegenerate anticommuting mass term is given by $m \in$ $\mathrm{GL}(n, \mathbb{R})$. This set has two connected components, hence there are two distinct phases. Note that the set $\mathrm{GL}(n, \mathbb{R})$ is homotopy equivalent to $R_{1}=O(n)$ (see Table 2 ); it provides the classification of systems with no symmetry for $d=1$ (cf. Table 1). We proceed with a more systematic approach. 


\section{CLASSIFICATION PRINCIPLES}

Concrete mathematical problems may be formulated for Dirac operators, band insulators, or more general systems. Let us set up the framework. We need to define a set of admissible Hamiltonians and some equivalence relation between them; the equivalence classes may then be called "phases". Continuous deformation, or homotopy is part of the equivalence definition, but it is not sufficient for a nice classification. A key idea in $K$-theory is that of stable equivalence: when comparing two objects, $X^{\prime}$ and $X^{\prime \prime}$, it is allowed to augment them by some object $Y$. We generally augment by a trivial system, i.e., a set of local, disjoint modes, like inner atomic shells. This corresponds to adding an extra flat band on an insulator. It may be the case that two systems cannot be continuously deformed one to the other, but such a deformation becomes possible after the augmentation. Thus, the topological classification of band insulators with an unlimited number of bands is simpler than in the case of two bands! Likewise, it is easier to classify Dirac operators if we do not impose any restriction on the size of gammamatrices. The final twist is that $K$-theory deals with differences between objects rather than objects themselves. Thus, we consider one phase relative to another.

We now give exact definitions for $d=0$ (meaning that the system is viewed as a single blob). The simplest case is where the particle number is conserved, but there are no other symmetries. A general free-fermion has this form:

$$
\hat{H}=\sum_{j, k} X_{j k} \hat{a}_{j}^{\dagger} \hat{a}_{k}
$$

where $X=\left(X_{j k}\right)$ is some Hermitian matrix representing electron hopping. Since we are interested in gapped systems, let us require that the eigenvalues of $X$ are bounded from both sides, e.g., $\Delta \leq\left|\varepsilon_{j}\right| \leq E_{\max }$. The following condition is slightly more convenient:

$$
\alpha \leq \varepsilon_{j}^{2} \leq \alpha^{-1},
$$

where $\alpha \leq 1$ is some constant. This class of matrices is denoted by $C_{0}(\alpha)$, and the corresponding Hamiltonians are called admissible. (Some locality condition will be needed in higher dimensions, but for $d=0$, this is it.)

The "spectral flattening" transformation, $X \mapsto \widetilde{X}=$ $\operatorname{sgn} X$ reduces admissible matrices to a special form, where all positive eigenvalues are replaced by +1 , all negative eigenvalues are replaced by -1 , and the eigenvectors are preserved. (The matrix element $\widetilde{X}_{j k}$ is, essentially, the equal-time Green function.) Such special matrices constitute the set

$$
C_{0}(\mathbf{1})=\bigcup_{0 \leq k \leq n} U(n) /(U(k) \times U(n-k)),
$$

where $n$ and $k$ are the matrix size and the numbers of -1 eigenvalues, respectively.
We write $X^{\prime} \approx X^{\prime \prime}$ (or $X^{\prime} \stackrel{\alpha}{\approx} X^{\prime \prime}$ to be precise) if $X^{\prime}$ and $X^{\prime \prime}$ are homotopic, i.e., can be connected by a continuous path within the matrix set $C_{0}(\alpha)$. It is easy to see that two matrices are homotopic if and only if they agree in size and have the same number of negative eigenvalues. For families of matrices, i.e., continuous functions from some parameter space $\Lambda$ to $C_{0}(\alpha)$, the homotopy classification is more interesting. For example, consider an integer quantum Hall system on a torus. The boundary conditions are described by two phases $(\bmod 2 \pi)$, therefore the parameter space is also a torus. This family of Hamiltonians is characterized by a nontrivial invariant, the first Chern number [28].

It is clear that $C_{0}(\alpha)$ can be contracted within itself to $C_{0}(1)$ since we can interpolate between the identity map and the spectral flattening: $X \mapsto f_{t}(X)$, where $t \in[0,1]$, $f_{0}(x)=x, f_{1}(x)=\operatorname{sgn} x$, and the function $f_{t}$ is applied to the eigenvalues of Hermitian matrix $X$ without changing the eigenvalues. Thus, $C_{0}(\alpha)$ is homotopy equivalent to $C_{0}(1)$, and we may use the latter set for the purpose of topological classification.

Let us consider this example (where $X$ is a single matrix or a continuous function of some parameters):

$$
Y_{0}=\left(\begin{array}{cc}
X & 0 \\
0 & -X
\end{array}\right) \approx\left(\begin{array}{cc}
0 & i I \\
-i I & 0
\end{array}\right)=Y_{1},
$$

The actual homotopy is $Y_{t}=\cos (t \pi / 2) Y_{0}+\sin (t \pi / 2) Y_{1}$. Note that $Y_{t}^{2}=1$ since $Y_{0}^{2}=Y_{1}^{2}=1$ and $Y_{0} Y_{1}=-Y_{1} Y_{0}$. Furthermore, $Y_{1}$ is homotopic to the matrix that consists of $\sigma^{z}$ blocks on the diagonal; such matrices will be regarded as trivial. This example shows that any admissible system $(X)$ is effectively canceled by its particle-hole conjugate $(-X)$, resulting in a trivial system. That is always true for free-fermion Hamiltonians, with any symmetry, in any dimension.

Equivalence between admissible matrices is defined as follows:

$$
X^{\prime} \sim X^{\prime \prime} \quad \text { if } \quad X^{\prime} \oplus Y \approx X^{\prime \prime} \oplus Y \text { for some } Y,
$$

where $\oplus$ means building a larger matrix from two diagonal blocks. Without loss of generality, we may assume that $Y$ is trivial. Indeed, if $X^{\prime} \oplus Y \approx X^{\prime \prime} \oplus Y$, then $X^{\prime} \oplus Y \oplus(-Y) \approx X^{\prime \prime} \oplus Y \oplus(-Y)$, and we have seen that $Y \oplus(-Y)$ is homotopic to a trivial matrix.

The difference class $d(A, B)$ of two same-sized matrices is represented by the pair $(A, B)$ up to this equivalence relation:

$$
\left(A^{\prime}, B^{\prime}\right) \sim\left(A^{\prime \prime}, B^{\prime \prime}\right) \quad \text { if } \quad A^{\prime} \oplus B^{\prime \prime} \sim A^{\prime \prime} \oplus B^{\prime} .
$$

Note that the the matrix sizes in different pairs need not be the same. Since $(A, B) \sim(A \oplus(-B), B \oplus(-B))$, it is sufficient to consider pairs where the second matrix is trivial. Thus, the equivalence class of $(A, B)$ is given by a 
single integer, $k=k(A)-k(B)$, where $k(\cdots)$ denotes the number of negative eigenvalues. Since $B$ is trivial, $k(B)$ equals half the matrix size, $n=2 s$. Hence, $k(A)=s+k$.

To characterize the difference between two families of matrices parametrized by $\Lambda$, one needs to consider functions from $\Lambda$ to the classifying space $C_{0}:^{3}$

$$
C_{0}=\bigcup_{k \in \mathbb{Z}} \lim _{s \rightarrow \infty} U(2 s) /(U(s+k) \times U(s-k)) .
$$

It is the same space as in Table 2. The Abelian group of difference classes ( $=$ homotopy classes of functions $\left.\Lambda \rightarrow C_{0}\right)$ is denoted by $K_{\mathbb{C}}^{0}(\Lambda)=\pi\left(\Lambda, C_{0}\right)$.

\section{SYMMETRIES AND CLIFFORD ALGEBRAS}

In this section, we complete the $d=0$ classification. Since the particle number is not generally conserved, we will use the Hamiltonian $H_{A}$ given by a real skewsymmetric matrix $A$ (see Eq. (2)). To generalize some arguments of the previous section, let us also define the trivial Hamiltonian: $\hat{H}_{\text {triv }}=\sum_{j}\left(\hat{a}_{j}^{\dagger} \hat{a}_{j}-\frac{1}{2}\right)=\hat{H}_{Q}$, where

$$
Q=\left(\begin{array}{ccccc}
0 & 1 & & & \\
-1 & 0 & & & \\
& & 0 & 1 & \\
& & -1 & 0 & \\
& & & & \ddots
\end{array}\right) .
$$

The eigenvalues of $A$ come in pairs $\left(+i \varepsilon_{j},-i \varepsilon_{j}\right)$, where $\varepsilon_{j}$ are positive and satisfy inequality (9). Replacing $A$ with $\widetilde{A}=-i \operatorname{sgn}(i A)$ takes $\varepsilon_{j}$ to 1 . The matrix $\widetilde{A}$ can be represented as $S Q S^{-1}$, where $S \in O(2 n)$. However, this representation is not unique since $S$ can be multiplied on the right by any orthogonal matrix that commutes with $Q$. Such matrices form a subgroup of $O(2 n)$ that may be identified with $U(n)$. Thus, the set of matrices $\widetilde{A}$ (i.e., real skew-symmetric matrices with $\pm i$ eigenvalues) is equal to $O(2 n) / U(n)$. Let us take the $n \rightarrow \infty$ limit by identifying $\widetilde{A}$ with $\widetilde{A} \oplus Q$ (where the size of $Q$ can be any even number). The result is listed in Table 2 as the classifying space $R_{2}$ :

$$
R_{2}=\lim _{n \rightarrow \infty} O(2 n) / U(n) .
$$

The set $R_{2}$ has two connected components, which are distinguished by the value of $\operatorname{sgn}(\operatorname{Pf} A)=\operatorname{Pf} \widetilde{A}=\operatorname{det} S= \pm 1$. The physical meaning of this invariant is the fermionic parity $(-1)^{\hat{N}}$ in the ground state, where $\hat{N}=\Sigma_{j} \hat{a}_{j}^{\dagger} \hat{a}_{j}$ is the particle number. Note that $\hat{N}$ is conserved $(\bmod 2)$.

\footnotetext{
${ }^{3}$ Here $\lim _{m \rightarrow \infty}$ is a so-called direct limit: the unitary cosets for smaller $m$ are mapped into ones for larger $m$.
}

The condition that $\hat{N}$ is conserved as an integer is equivalent to a $U(1)$ symmetry. In this case, the creationannihilation expression of Hamiltonian (2) should not contain terms like $\hat{a}_{j} \hat{a}_{k}$ or $\hat{a}_{k}^{\dagger} \hat{a}_{j}^{\dagger}$. This is a good point to note that the approach based on free-fermion Hamiltonians is fundamentally incomplete since it cannot distinguish between the full $U(1)$ group and its $\mathbb{Z}_{4}$ subgroup, which is generated by the transformation $\hat{a}_{j} \mapsto i \hat{a}_{j}$. Let us assume for a moment that the actual symmetry is $\mathbb{Z}_{4}$. Then terms like $\hat{a}_{1} \hat{a}_{2} \hat{a}_{3} \hat{a}_{4}$ are allowed in principle, but not in a free-fermion Hamiltonian. Therefore topological invariants of noninteracting systems may not be preserved in the presence of interactions. In the following example, the number of particle-occupied states changes by 4 by a continuous path through an interacting phase:

$$
\hat{H}(t)=\cos (\pi t) \sum_{j=1}^{4} \hat{a}_{j}^{\dagger} \hat{a}_{j}+\sin (\pi t)\left(\hat{a}_{1} \hat{a}_{2} \hat{a}_{3} \hat{a}_{4}+\text { h.c. }\right) .
$$

Note that the ground state remains non-degenerate for all values of $t$. On the other hand, a homotopy like that is only possible if the interaction term exceeds the energy gap at some point. Thus, the noninteracting topological classification is generally stable to weak interactions, but not to strong ones. In the $U(1)$ case, it is absolutely stable though (at least, for $d=0$ ). We now set this discussion aside and proceed with the noninteracting case.

It is easy to see that the Hamiltonian (2) is $U(\mathbf{1})$ invariant if and only if the matrix $A$ commutes with $Q$ (see Eq. (14)). Another possible symmetry is timereversal invariance. It can be expressed by an antiunitary operator $\hat{T}$ acting in the Fock space; this action is defined as follows:

$$
\hat{T} i \hat{T}^{-1}=-i, \quad \begin{array}{ll}
\hat{T} \hat{a}_{j \uparrow} \hat{T}^{-1}=\hat{a}_{j \downarrow}, \quad \hat{T} \hat{a}_{j \uparrow}^{\dagger} \hat{T}^{-1}=\hat{a}_{j \downarrow}^{\dagger}, \\
\hat{T} \hat{a}_{j \downarrow} \hat{T}^{-1}=-\hat{a}_{j \uparrow}, \quad \hat{T} \hat{a}_{j \downarrow}^{\dagger} \hat{T}^{-1}=-\hat{a}_{j \uparrow}^{\dagger}
\end{array}
$$

Converting $\hat{a}_{1 \uparrow}, \hat{a}_{1 \uparrow}^{\dagger}, \hat{a}_{1 \downarrow}, \hat{a}_{1 \downarrow}^{\dagger}, \ldots$ into $\hat{c}_{1}, \hat{c}_{2}, \hat{c}_{3}, \hat{c}_{4}, \ldots$, we obtain a relation of the form $\hat{T} \hat{c}_{m} \hat{T}^{-1}=\sum_{l} T_{l m} \hat{c}_{l}$, where the matrix $T$ consists of $4 \times 4$ blocks:

$$
T=\left(\begin{array}{ccccc}
0 & 0 & -1 & 0 & \\
0 & 0 & 0 & 1 & \\
1 & 0 & 0 & 0 & \\
0 & -1 & 0 & 0 & \\
& & & & \ddots
\end{array}\right)
$$

The $\hat{T}$-invariance of the Hamiltonian is equivalent to the condition $T A=-A T$.

Let us describe a common algebraic structure that is applicable to three symmetry types: no symmetry, $T$ only, and $T$ and $Q$. First, note these identities:

$$
T^{2}=Q^{2}=-1, \quad T Q=-Q T .
$$


It is convenient to introduce some new notation: $e_{1}=T$, $e_{2}=Q T$. Note that $e_{2}$ anticommutes with $A$ if both the $T$ and $Q$ symmetries are present. Let us also use $\widetilde{A}=-i \operatorname{sgn}(i A)$ instead of $A$. Then we have the following characterization:

No symmetry: $\quad \widetilde{A}^{2}=-1$;

$T$ only: $\quad e_{1}^{2}=\widetilde{A}^{2}=-1, \quad e_{1} \widetilde{A}=-\widetilde{A} e_{1} ;$

$T$ and $Q: \quad e_{1}^{2}=e_{2}^{2}=\widetilde{A}^{2}=-1$,

$$
e_{1} e_{2}=-e_{2} e_{1}, \quad e_{j} \widetilde{A}=-\widetilde{A} e_{j} \quad(j=1,2) .
$$

The pattern is pretty obvious. We have $p$ predefined matrices $e_{1}, \ldots, e_{p} \quad(p=0,1,2)$ satisfying Clifford algebra relations (see exact definition below) and look for all possible choices of another Clifford generator $e_{p+1}=\widetilde{A}$.

The (real) Clifford algebra Ciff $^{p, q}$ is generated by elements $e_{1}, \ldots, e_{p+q}$ satisfying these relations: ${ }^{4}$

$$
\begin{gathered}
e_{1}^{2}=\ldots=e_{p}^{2}=-1, \quad e_{p+1}^{2}=\ldots=e_{p+q}^{2}=1, \\
e_{j} e_{k}=-e_{k} e_{j} \quad(j \neq k) .
\end{gathered}
$$

All Clifford algebras can be described in terms of the 3 simple algebras with real coefficients: $\mathbb{R}$ (real numbers), $\mathbb{C}$ (complex numbers), and $\mathbb{H}$ (quaternions). For example, Ciff ${ }^{0,1}$ is isomorphic to $\mathbb{R} \oplus \mathbb{R}$ since it consists of linear combinations of two complementary projectors, $\frac{1}{2}\left(1 \pm e_{1}\right)$. The algebra $\mathrm{Ciff}^{1,0}$ can be identified with $\mathbb{C}$ by mapping the negative generator $e_{1}$ to $i$. Furthermore, $\mathrm{Ciff}^{0,2} \cong \mathrm{Ciff}^{1,1} \cong \mathbb{R}(2)$ (the algebra of real $2 \times 2$ matrices where the Clifford generators are mapped to $\sigma^{z}, \sigma^{x}$ or to $\sigma^{z}, i \sigma^{y}$, respectively), and $\operatorname{Ciff}^{2,0} \cong \mathbb{H}$. For more details on Clifford algebras and their use in $K$-theory, see Refs. [26, 25].

In the problem at hand, the Clifford generators act in the mode space. 5 Thus, we deal with Clifford algebra representations such that $e_{1}, \ldots, e_{p}$ and $e_{p+1}, \ldots, e_{p+q}$ are represented by real skew-symmetric and real symmetric matrices, respectively. To classify free-fermion Hamiltonians, we consider representations of $\operatorname{Ciff}^{p+1,0}$ with fixed action of $e_{1}, \ldots, e_{p}$; we call that the "Clifford extension problem with $p$ negative generators".

For technical reasons, it is convenient to reformulate the problem in terms of positive generators. To this end, we will employ the isomorphism Ciff $^{0, p+2} \cong$ Ciff $^{p, 0} \otimes \mathbb{R}(2)$, which may be defined as follows:

$$
\begin{array}{cc}
e_{j} \mapsto e_{j}^{\prime} \otimes\left(i \sigma^{y}\right) & \text { for } j=1, \ldots, p \\
e_{p+1} \mapsto I \otimes \sigma^{z}, & e_{p+2} \mapsto I \otimes \sigma^{x} .
\end{array}
$$

\footnotetext{
4 An alternative notation is also used, where the positive generators $\left(e_{j}^{2}=1\right)$ are listed first and the parameters $p$ and $q$ are swapped.

5 In comparison, the Majorana operators $\hat{c}_{l}$ generate a (complex) Clifford algebra acting in the Fock space.
}

Representations of the algebra $\mathscr{A} \otimes \mathbb{R}(n)$ (for any $\mathscr{A}$ ) have very simple structure, namely, $E \otimes \mathbb{R}^{n}$, where the first factor is some representation of $\mathscr{A}$ and the second comes with the standard action of the matrix algebra $\mathbb{R}(n)$. Thus, $\mathscr{A}$ and $\mathscr{A} \otimes \mathbb{R}(n)$ have the same representation theory (i.e., their representations are in a natural oneto-one correspondence); such algebras are called Morita equivalent. Up to Morita equivalence, $\mathrm{Ciff}^{p, q}$ only depends on $p-q \bmod 8$.

We conclude that the classification of free-fermion Hamiltonians with $p$ negative Clifford symmetries is equivalent to the extension problem with $q=p+2$ positive generators. That is to say, we need to find all possible actions of $e_{q+1}\left(e_{q+1}^{2}=1\right)$ if the action of $e_{1}, \ldots, e_{q}$ is fixed. In $K$-theory, the problem is formulated in terms of difference objects $(E, F, w)$, where $E, F$ are representations of $\mathrm{Ciff}^{0, q+1}$ and $w$ is a linear orthogonal map that identifies them as $\mathrm{Ciff}^{0, q}$ representations, see [25]. Without loss of generality, we may fix $F$ to be a sum of several copies of the regular representation (which corresponds to a trivial Hamiltonian) and $w$ the identity map. Such difference objects form the classifying space $R_{q}$ (see Table 2). The Abelian group of equivalence classes of difference objects parametrized by $\Lambda$ is $K_{\mathbb{R}}^{0, q}(\Lambda)=\pi\left(\Lambda, R_{q}\right)$. It is isomorphic to the conventional real $K$-group $K_{\mathbb{R}}^{-q}(\Lambda)$, which is also denoted by $K O^{-q}(\Lambda)$. In the special case where $\Lambda=$ pt (a single point), we get $K_{\mathbb{R}}^{-q}(\mathrm{pt})=\pi_{0}\left(R_{q}\right)$.

\section{CLASSIFICATION FOR ARBITRARY $d$}

We begin with a short summary, focusing on the symmetry classes that correspond to real $K$-theory. It is natural to distinguish three cases:

1. Continuous free-fermion Hamiltonians are classified by $\widetilde{K}_{\mathbb{R}}^{-q}\left(\bar{S}^{d}\right)=\pi_{0}\left(R_{q-d}\right)$, where $\bar{S}^{d}$ represents the momentum space (see below). Sufficient insight can be gained by considering Dirac operators. This setting is actually more general than one might expect: gapped Hamiltonians in the momentum space are topologically equivalent to nondegenerate mass terms that anticommute with a fixed Dirac operator. Long-range disorder may be described by textures of the mass term varying in space, i.e., continuous functions $M: \mathbb{R}^{d} \rightarrow R_{q-d}$.

2. Band insulators are characterized by the momentum space $\overline{\mathbb{T}}^{d}$, hence the classification is given by $K_{\mathbb{R}}^{-q}\left(\overline{\mathbb{T}}^{d}\right)$. This Abelian group includes $\pi_{0}\left(R_{q-d}\right)$ as a direct summand, but there is some extra piece (cf. "weak topological insulators").

3. Arbitrary local discrete systems under the energy gap or localization condition. ("Local" means that 
the electron hopping is short-ranged. The gap condition is stronger than the localization, but the problem for the localized case can be reduced to that for the gapped case.) Realizations of short-range disorder fall into this category. The classification of such general systems is exactly the same as for Dirac operators, due to the following

Theorem: Any gapped local free-fermion Hamiltonian in $\mathbb{R}^{d}$ is equivalent to a texture.

(That is the key technical result, but I cannot explain it in any detail in such a short note.) Discrete systems on a compact metric space $L$ are classified by the $K$-homology group $K_{q}^{\mathbb{R}}(L)$.

\section{Continuous systems and Dirac operators}

The Hamiltonian of a translationally invariant systems can be written in the momentum representation:

$$
\hat{H}=\frac{i}{4} \sum_{\mathbf{p}} \sum_{j, k} A_{j k}(\mathbf{p}) \hat{c}_{-\mathbf{p}, j} \hat{c}_{\mathbf{p}, k}
$$

where $j$ and $k$ refer to particle flavors. The matrix $A(\mathbf{p})$ is skew-Hermitian but not real; it rather satisfies the condition $A_{j k}(\mathbf{p})^{*}=A_{j k}(-\mathbf{p})$. By abuse of terminology, such matrix-valued functions are called "functions from $\overline{\mathbb{R}}^{d}$ to real skew-symmetric matrices", where $\overline{\mathbb{R}}^{d}$ is the usual Euclidean space with the involution $\mathbf{p} \leftrightarrow-\mathbf{p}$ (cf. [29]). The symmetry is defined by some Clifford generators represented by real matrices whose action does not depend on p. As described in the previous section, we can turn negative generators to positive and replace $A(\mathbf{p})$ by another Clifford generator $e_{q+1}$ (p). While the matrices $e_{1}, \ldots, e_{q}$ are real symmetric, $e_{q+1}$ is Hermitian and satisfies the condition $e_{q+1}(\mathbf{p})^{*}=e_{q+1}(-\mathbf{p})$.

A reasonable classification can be developed when the asymptotics of $A(\mathbf{p})$ for $|\mathbf{p}| \rightarrow \infty$ is fixed. We may identify the infinity in the momentum space with the boundary of a large ball, $\partial \bar{B}^{d}$. Thus, the difference between two phases may be characterized by an element of the relative $K$-group

$$
K_{\mathbb{R}}^{0, q}\left(\bar{B}^{d}, \partial \bar{B}^{d}\right)=\widetilde{K}_{\mathbb{R}}^{0, q}\left(\bar{S}^{d}\right) \cong \pi_{0}\left(R_{q-d}\right) .
$$

Here we have used the isomorphism [25]

$$
\widetilde{K}_{\mathbb{R}}^{p, q}(X) \cong \widetilde{K}_{\mathbb{R}}^{0}\left(S^{r} X\right) \quad(r=q-p \bmod 8),
$$

and the $(1,1)$ periodicity [29]:

$$
\widetilde{K}_{\mathbb{R}}^{0}(S \bar{S} X) \cong \widetilde{K}_{\mathbb{R}}^{0}(X),
$$

where $S$ denotes the suspension.

The group $\pi_{0}\left(R_{q-d}\right) \cong K_{\mathbb{R}}^{d, q}(\mathrm{pt})$ on the right-hand side of Eq. (20) has a concrete physical interpretation. It classifies the nondegenerate mass terms $M$ in the real self-adjoint Dirac operator $D=\sum_{a} \gamma_{a} \partial_{a}+M$, where $\gamma_{a}$ are skew-symmetric, $M$ is symmetric, and

$$
\gamma_{a} \gamma_{b}+\gamma_{b} \gamma_{a}=-\delta_{a b}, \quad \gamma_{a} M=-M \gamma_{a}
$$

(Replacing $M$ with $\widetilde{M}=\operatorname{sgn} M$, we can achieve that $M^{2}=1$.) In addition, we assume that $\gamma_{1}, \ldots, \gamma_{d}$ and $M$ anticommute with the symmetry generators $e_{1}, \ldots, e_{q}$. Thus, the gamma-matrices play the role of Clifford symmetries with opposite sign; they effectively cancel the actual symmetries. Note that those new "symmetries" do not entail any conservation laws. Our argument only implies that any continuous spectrum is equivalent (up to an augmentation and homotopy) to a Dirac spectrum that has the additional symmetries.

\section{Discrete systems}

Let us consider the Hamiltonian (2), where each mode $j$ is associated with a site, or point $\mathbf{r}_{j}$ in the real space. There may be several modes per site; symmetries (if any) act independently on each site. We assume that the Hamiltonian is $r$-local (i.e., $A_{j k}=0$ if the distance between $\mathbf{r}_{j}$ and $\mathbf{r}_{k}$ is greater than $r$ ) and that it is $\alpha$-gapped (i.e., the eigenvalues $\varepsilon_{j}$ of $i A$ satisfy inequality (9)). Under these conditions, the matrix element $\widetilde{A}_{j k}$ decays very fast as the distance between $j$ and $k$ goes to infinity, which is a sign of localization. Conversely, if we start with the matrix $\widetilde{A}$ (such that $\widetilde{A}^{2}=-1$ and $\widetilde{A}_{j k}$ decays fast enough) and set all the elements for $\left|\mathbf{r}_{j}-\mathbf{r}_{k}\right|>r^{\prime}$ to zero, we will obtain a gapped local matrix. Both transformations can be done continuously, which roughly shows that the set of localizing Hamiltonians is contractible within itself to the set of gapped Hamiltonians (up to a change of controlling parameters). Thus, we may stick with the gapped case without any loss of generality.

Using the standard trick, we replace $A$ with a real symmetric matrix $X$ that is $r$-local, $\alpha$-gapped, and anticommutes with $q$ positive Clifford symmetries. The abovementioned theorem pertains to such matrices. Here, we only discuss it at the physical level. The texture corresponding to the matrix $X$ is constructed algorithmically, albeit in a contrived fashion. The procedure is local, with a characteristic radius $l=c r$, where $c$ depends on $d$ and $\alpha$. The number of Dirac modes needed is $l^{d}$ (for localized systems, it's the localization volume). To calculate $M(\mathbf{r})$, we only look at the $l$-neighborhood of point $\mathbf{r}$, and $M$ doesn't vary much at distances smaller than $l$. We may then discretize the Dirac operator on a fine grid, with $\gamma_{a}$ and $M$ rescaled properly so as to keep the $\alpha$ parameter fixed. Thus, we obtain an $r^{\prime}$-local, $\alpha$-gapped matrix $X^{\prime}$, where $r^{\prime}$ is arbitrary small. The equivalence between $X$ and $X^{\prime}$ involves an augmentation and a homotopy, where 
$r$ may increase by a constant factor before it shrinks down to $r^{\prime}$.

This theorem implies that the boundary between two phases must carry some gapless modes. Indeed, each phase may be characterized by the mass term $M(\mathbf{r})$ computed at any point away from the boundary. Since the phases are different, the two mass terms, $M\left(\mathbf{r}_{1}\right), M\left(\mathbf{r}_{2}\right)$ belong to different connected components of the classifying space. But if the boundary between the phases were gapped, we could make the whole system into a continuous texture, and thus $M\left(\mathbf{r}_{1}\right)$ and $M\left(\mathbf{r}_{2}\right)$ would belong to the same component - a contradiction.

A gapped local system on a compact metric space $L$ (say, a manifold with or without boundary) is characterized by a $K$-homology class $\xi \in K_{q}^{\mathbb{R}}(L)$, where $q$ is defined $(\bmod 8) . K$-homology (see e.g. [30]) and the related noncommutative geometry [31] are advanced subjects, but the basic intuition is rather simple. Let us consider systems with no symmetry $(q=2)$ on the two-dimensional torus, $L=\mathbb{T}^{2}$. Such systems are trivially characterized by the number of fermions in the ground state, $\xi_{0} \in \mathbb{Z}_{2}$. Now imagine a closed Majorana chain winding around the torus. It defines a homology class $\xi_{1} \in H_{1}\left(\mathbb{T}^{2} ; \mathbb{Z}_{2}\right)$, which is a topological invariant for gapped local systems. It can be measured by cutting the torus along some cycle $c$ and counting edge modes $(\bmod 2)$. Or one can flip the sign of all matrix elements $A_{j k}$ spanning across the cut and see how $\xi_{0}$ changes: $\xi_{1}(c)=\xi_{0}(+) \xi_{0}(-)$. If the torus is filled with a $p_{x}+i p_{y}$ superconductor, the system has a nontrivial twodimensional invariant, $\xi_{2} \in \mathbb{Z}$. But if $\xi_{2}$ is odd, then the properties of the $1 \mathrm{D}$ invariant change: $\xi_{1}$ is not a homology class, but rather, a spin structure. Indeed,

$$
\xi_{0}(++) \xi_{0}(+-) \xi_{0}(-+) \xi_{0}(--)=(-1)^{\xi_{2}},
$$

where \pm refers to the sign of matrix elements across two basis cycles. In general, the definition of lowdimensional invariants (except in dimension 0 ) depends on the higher-dimensional ones. ${ }^{6}$ The $K$-homology class includes all.

\section{Band insulators}

The main difference from continuous systems is that the momentum space is $\bar{T}^{d}$. Since there is no need to fix the spectrum at infinity, the classification is given by the absolute $K$-group $K_{\mathbb{R}}^{0, q}\left(\bar{T}^{d}\right) \cong K_{\mathbb{R}}^{-q}\left(\bar{T}^{d}\right)$. The band structure analysis in Refs. $[2,5,8]$ and others offers a concrete view of that group in certain cases. Unfortunately,

\footnotetext{
${ }^{6}$ The term "invariant" is used in a sloppy way, but one can rigorously define the range of $\xi_{s}$, assuming that $\xi_{s+1}=\ldots=\xi_{d}=0$. It is the Abelian group $E_{s, q-s}^{\infty}$ of the $K$-homology spectral sequence.
}

the momentum space picture is non very intuitive. To understand and calculate the group $K_{\mathbb{R}}^{-q}\left(\bar{T}^{d}\right)$, we relate it to $K$-homology of the real-space torus by means of the Baum-Connes isomorphism for $\mathbb{Z}^{d}$ (a $K$-theory analogue of the Fourier transform). Then we apply the Poincare duality. Thus,

$$
\begin{aligned}
K_{\mathbb{R}}^{-q}\left(\overline{\mathbb{T}}^{d}\right) \cong K_{q}^{\mathbb{R}}\left(\mathbb{T}^{d}\right) \cong K_{\mathbb{R}}^{d-q}\left(\mathbb{T}^{d}\right) \\
\cong \pi_{0}\left(R_{q-d}\right) \oplus \widetilde{K}_{\mathbb{R}}^{d-q}\left(\mathbb{T}^{d}\right)
\end{aligned}
$$

The first term is the same as before, but the last one is new. It further splits, though not canonically:

$$
\widetilde{K}_{\mathbb{R}}^{d-q}\left(\mathbb{T}^{d}\right) \cong \bigoplus_{s=0}^{d-1}\left(\begin{array}{l}
d \\
s
\end{array}\right) \pi_{0}\left(R_{q-s}\right) .
$$

For 3D $T$-invariant insulators, i.e., $d=3, q=4$, we get:

$$
\widetilde{K}_{\mathbb{R}}^{-1}\left(\mathbb{T}^{3}\right) \cong \mathbb{Z} \oplus 3 \mathbb{Z}_{2}
$$

The $\mathbb{Z}$ term is the number of (Kramers degenerate) valence bands, whereas $3 \mathbb{Z}_{2}$ pertains to "weak topological insulators".

\section{THE EFFECT OF INTERACTION}

Topological properties of gapped local free-fermion systems are mostly understood. The big open question is how the classification is changed by interactions, e.g., whether different free-fermion phases can be deformed one to another through an interacting phase without closing the gap. In some cases, e.g., the integer quantum Hall effect and chiral 2D superconductors, the topological invariants are related to physical properties that are welldefined in the presence of interactions (namely, the Hall conductivity and the chiral central charge, which determines the edge energy current $[32,16])$. The Kramers degeneracy analysis of vortex-bound states demonstrates the stability of 2D topological insulators [2, 33] and $\left(p_{x}+i p_{y}\right) \uparrow+\left(p_{x}-i p_{y}\right) \downarrow$ superconductors [23].

However, the free-fermion classification is unstable for 1D systems with the unusual $T$ symmetry: $\hat{T}^{2}=1$ instead of $\hat{T}^{2}=(-1)^{\hat{N}}$. For a concrete model, consider the Majorana chain and its variations, where $\hat{T}$ acts on odd sites by $\hat{T} \hat{c}_{j} \hat{T}^{-1}=-\hat{c}_{j}$ so that terms like $i \hat{c}_{j} \hat{c}_{k}$ are only allowed between sites of different parity. In the free-fermion setting, this symmetry is described by one positive Clifford generator, hence $p=-1, q=p+2=$ 1 , and for $d=1$ we get a topological invariant $k \in$ $\pi_{0}\left(R_{q-d}\right)=\mathbb{Z}$. For example, the usual phase transition in 8 parallel Majorana chains is characterized by $k=8$. But in this particular case, the two phases are actually connected through an interacting phase [34]. 


\section{ACKNOWLEDGMENTS}

I am grateful to Andreas Ludwig and Shinsey Ryu for teaching me about ${ }^{3} \mathrm{He}-B$ and $\left(p_{x}+i p_{y}\right) \uparrow+\left(p_{x}-i p_{y}\right) \downarrow$ and helping to fit these phases into the periodic table. I also thank John Preskill, Michael Freedman, John Roe, Charles Kane, and Grigori Volovik for stimulating discussions. This research is supported in part by NSF under grant No. PHY-0456720.

\section{REFERENCES}

1. C.L. Kane and E.J. Mele, Phys. Rev. Lett. 95, 226801 (2005).

2. C.L. Kane and E.J. Mele, Phys. Rev. Lett. 95, 146802 (2005).

3. B.A. Bernevig, T.L. Hughes and S.-C. Zhang, Science 314, 1757 (2006).

4. M. Konig, S. Wiedmann, C. Brune, A. Roth, H. Buhmann, L.W. Molenkamp, X.-L. Qi and S.-C. Zhang, Science 318, 766 (2007).

5. J.E. Moore and L. Balents, Phys. Rev. B 75, 121306 (2007).

6. R. Roy, cond-mat/0607531

7. R. Roy, arXiv:0803.2868.

8. L. Fu, C.L. Kane and E.J. Mele, Phys. Rev. Lett. 98, 106803 (2007).

9. K. Sengupta et al, Phys. Rev. B 63, 144531 (2001).

10. L. Fu and C.L. Kane, Phys. Rev. B 76, 144532 (2007).

11. D. Hsieh et al, Nature 452, 970 (2008).

12. S.B. Bravyi and A.Y. Kitaev, quant-ph/9811052.

13. D.J. Thouless, Phys. Rev. Lett. 49, 405 (1982).

14. A. Kitaev, cond-mat/0010440.

15. N. Read and D. Green, Phys. Rev. B 61, 10267 (2000).

16. A. Kitaev, Annals of Physics 321, 2 (2006).

17. J. Bellissard, in Lecture Notes in Physics 257 (1986).

18. J.E. Avron, R. Seiler and B. Simon, Phys. Rev. Lett. 65 , 2185 (1990).

19. J. Bellisard, A. van Elst, and H. Schulz-Baldes, J. Math. Phys. 35, 5373 (1994).

20. A. Altland and M.R. Zirnbauer, Phys. Rev. B 55, 1142 (1997).

21. A.P. Schnyder et al, Phys. Rev. B 78, 195123 (2008).

22. X.-L. Qi, T.L. Huges and S.-C. Zhang, Phys. Rev. B 79, 195424 (2008).

23. X.-L. Qi et al, arXiv:0803.3614.

24. P. Horava, Phys. Rev. Lett. 95, 16405 (2005).

25. M. Karoubi, K-theory: an introduction, Springer-Verlag, 1978.

26. M.F. Atiyah, R. Bott and A. Shapiro, Topology 3, 38 (1964).

27. L. Fu and C.L. Kane, arXiv:0804.4469.

28. Q. Niu, D.J. Thouless and Y.-S. Wu, Phys. Rev. B 31, 3372 (1985).

29. M.F. Atiyah, The Quarterly Journal of Mathematics 17, 367 (1966).

30. N. Higson and J. Roe, Analytic K-homology, Oxford University Press, New York, 2000.

31. A. Connes, Noncommutative geometry, Academic Press, San Diego, 1994.
32. C.L. Kane and M.P.A. Fisher, title=Quantized thermal transport in the fractional quantum Hall effect, author=Kane, C.L. and Fisher, M.P.A., Phys. Rev. $B$ 55, 15832 (1997).

33. L. Fu and C.L. Kane, Phys. Rev. B 74, 195312 (2006).

34. L. Fidkowski. and A. Kitaev, in preparation. 